\title{
Patrones de índice verde normalizado (IVN) en pastizales de la provincia de Buenos Aires: su asociación con comunidades vegetales y suelos
}

\author{
Pablo VázQuez ${ }^{1, \otimes}$; Mirta Calandroni²; Fabián Cabria ${ }^{2}$; Mónica Agnusdei ${ }^{3}$ \& María \\ DEL C. ROJAS ${ }^{1}$
}

1.Estación de Experimentación Agropecuaria Guillermo Covas, INTA, La Pampa, Argentina. 2. Facultad de Ciencias Agrarias Balcarce. Universidad Nacional de Mar del Plata, Argentina. 3. Estación de Experimentación Agropecuaria Balcarce, Argentina.

\begin{abstract}
Resumen. A partir de información satelital histórica (MODIS 2000-2010), se identificaron patrones anuales de índice verde normalizado (IVN) pertenecientes a áreas ocupadas por pastizales o pasturas de la Pampa Deprimida, y se asoció su distribución espacial a la de diferentes comunidades vegetales y de suelos mapeados a escala 1:25000. Se identificaron doce patrones de IVN (PA). Nueve de ellos presentaron dos estaciones de crecimiento y cuatro presentaron una única estación. El inicio de la primera estación de crecimiento fue determinado en agosto, con un valor máximo del IVN en octubre, mientras que la segunda estación de crecimiento presentó su valor máximo en marzo. El valor basal de IVN fue observado durante el período junio-julio, coincidente con el período de reposo de la vegetación. El ajuste del mapa de suelos existente 1:50000 a un mayor grado de resolución (1:25000) a partir del mapa de riesgo hídrico y el modelo digital de terreno SRTM 90 m fue satisfactorio para la identificación de subgrupos de suelos. Los PA tuvieron una distribución espacial compatible con las comunidades observadas a campo y con las propiedades edáficas mapeadas a escala detallada. Las comunidades vegetales tienen la capacidad de adaptarse a situaciones edáficas diferentes, borrando el efecto suelo, salvo en sitios con severas restricciones edáficas o muy disturbados.
\end{abstract}

[Palabras clave: biometría, Pampa Deprimida, mapa de suelos]

\begin{abstract}
Aвstract. Normalized Difference Vegetation Index (NDVI) patterns in grasslands of the Buenos Aires province: their association to plant communities and soils: From historical satellite data (MODIS 2000-2010), annual NDVI patterns belonging to areas of the flooding Pampas occupied by grassland or pastures were identified, associating their spatial distribution to different plant communities and soils mapped at 1:25000 scale. Twelve green index normalized patterns (PA) were identified, nine of them with two growing seasons and four PA with only one. The beginning of the regional photosynthetic activity was recorded in August, with a first NDVI peak in October and the second growing season with a NDVI peak in March. Basal NDVI was observed during the June-July period, coincident with vegetation dormancy. The adjustment of soils maps scale 1:50000 to a major degree of resolution (1:25000) with application of a flooding risk map and the SRTM $90 \mathrm{~m}$ digital elevation model was satisfactory for soils subgroups identification. PA had a spatial distribution compatible with observed grassland communities in the field and with soil properties mapped at detailed scale. Grassland communities seem to have de ability to adapt themselves to different soil situations, smoothing soil effects at detailed scales, except in areas with severe soil restrictions or very disturbed.
\end{abstract}

[Keywords: biometrics, flooding Pampas, soil map]

\section{INTRODUCCIÓN}

La distribución de comunidades vegetales y su asociación con el tipo de suelo se ha utilizado en todo el mundo para interpretar la variación estacional y el potencial de producción primaria de los pastizales (Burkart et al. 1990; Tiezen et al. 1997; Nouvellon et al. 2001; Batista et al. 2005). La teledetección ha facilitado el estudio de la dinámica y distribución espacial de la vegetación a partir de la interpretación de estimadores de la actividad fotosintética, como son los índices verdes (Rouse et al. 1974). También se desarrollaron algoritmos que permiten

Editora asociada: Roxana Aragón

$\triangle$ vazquez.pablo@inta.gob.ar estimar parámetros biométricos derivados de las curvas estacionales de índice verde, a partir de los cuales pueden clasificarse las cubiertas vegetales por comportamiento homogéneo (Goetz 1997; Tiezen et al. 1997; Boles 2004; Jönsson \& Ecklhund 2003).

Existen varios tipos de zonificación para el estudio de variables biofísicas a escala global, pero no siempre logran responder a las necesidades locales (Mattar et al. 2008). El patrón espacial presente en las comunidades vegetales a diferentes escalas de análisis indicaría el grado de control que ejerce el ambiente sobre su estructura y composición, 
considerándose el tamaño de las unidades (parches) y el tamaño del sistema (Allen et al. 1984; Turner \& Gardner 1991; Perelman et al. 2001). En la medida que los estudios se realizan a escalas más amplias, los patrones regionales se vuelven más robustos que los locales. Por otra parte, las variaciones ambientales que operan a escala detallada pueden determinar patrones de comportamiento vegetal tan fuertes que pueden observarse a escalas regionales.

En la Pampa Deprimida se han realizado varios estudios sobre la composición florística de ambientes a escala regional y de paisaje (Vervoorst 1967; León et al. 1979; Batista et al. 1988; Burkart et al. 1990; 1998). Estos trabajos fueron hechos durante un período en el que la actividad ganadera era extensiva, con cargas que no superaban los 0.7 equivalentes vaca por hectárea (Bocchetto 1979), y coexistía con comunidades vegetales en su mayoría autóctonas (Perelman et al. 2001). Se describieron como máximo 17 comunidades, lo cual confirmó que la mayor heterogeneidad en composición florística fue observada a escala de paisaje (Verboorst 1967; Perelman et al. 2001). Burkart et al. (2005) definieron un mapa de distribución fitosociológica potencial considerando como información complementaria el mapa de suelos de la provincia de Buenos Aires a escala 1:500000. El concepto de suelo-paisaje sostiene que si uno conoce las relaciones entre cada uno de los suelos y su entorno se puede inferir el tipo de suelo presente en cada sitio. Dokuchaev (1886) fue el primero en reconocer que el suelo obedece a leyes deterministas y que su estado actual se asocia a diferentes fenómenos de la naturaleza. Consideró al suelo como un ente complejo, estructurado y dotado de regímenes cíclicos (diarios, mensuales y anuales), cuyos caracteres están en relación con los factores de diferenciación: rocas, clima, topografía, seres vivos y edad. La actualización de la cartografía edáfica es dificultosa debido a los costos operativos que implica un relevamiento de suelos. La teledetección ha facilitado, en este aspecto, identificar geoformas y paisajes mediante la elaboración de modelos digitales de terreno (MDT) (Burrough et al. 2000; Zhu et al. 2001). Esto permitiría mejorar la cartografía edáfica existente $\mathrm{y}$, por ende, la delimitación de paisajes y ambientes.

El objetivo de este trabajo fue identificar patrones de IVN en áreas ocupadas por pastizales y pasturas de la Pampa Deprimida, caracterizar su biometría y relacionar su distribución con las comunidades vegetales del área y el tipo de suelo a escala detallada.

\section{MATERIALES y MÉTODOS}

La Pampa Deprimida es una llanura extensa y anegable que ocupa 7000000 ha de la provincia de Buenos Aires, Argentina. Su clima es templado húmedo, con una temperatura media anual de 13.8 ${ }^{\circ} \mathrm{C}$ en el sur y $15.9^{\circ} \mathrm{C}$ en el norte. La precipitación media anual es $\sim 900 \mathrm{~mm}$.

Las imágenes satelitales utilizadas fueron provistas por el proyecto Earth Observation System (EOS) de la NASA (1999), disponibles en frp:/ /e4ft101u.ecs.nasa.gov/.

\section{Determinación de PA}

La identificación de PA fue realizada a partir del análisis de la serie temporal de IVN quincenales MODIS TERRA, producto MOD13Q1 (series de IVN con resolución espacial de $250 \mathrm{~m}$ ), período 2000-2009 (230 imágenes). Se eliminaron del análisis las áreas destinadas a agricultura y urbanizadas (Vázquez et al. 2009). Basados en una experiencia anterior (Vázquez et al. 2013), la identificación de PA en la Pampa Deprimida se realizó aplicando la técnica de análisis multivariada KMEANS (Mc Queen 1967) sobre la serie temporal promedio de IVN del producto MOD13Q1. Dado un conjunto inicial de $\mathrm{k}$ centroides $\mathrm{m} 1(1), \ldots, \mathrm{mk}(1)$, el algoritmo utiliza una técnica iterativa para asignar cada observación al grupo con la media más cercana (es decir, la partición de las observaciones de acuerdo con el diagrama de Voronoi generado por los centroides). El objetivo del algoritmo de KMEANS es reducir al mínimo la variabilidad (la suma de las distancias al cuadrados) entre cada píxel y su centro de clúster asignado. El modelo fue restringido para que no genere grupos con una superficie inferior a $1 \%$ de la superficie total (70000 ha). Por lo tanto, esos píxeles son reasignados considerando la menor distancia euclidiana hacia el centroide del clúster más próximo. El procesado de las imágenes satelitales se realizó mediante el programa IDRISI TAIGA (@ 2009 Clark Labs, Clark University, USA).

\section{Determinación de curvas de IVN y parámetros biométricos típicos para los diferentes $P A$}

Se ajustaron las curvas típicas de IVN de cada PA aplicando la función Savitsky-Golay, Timesat 3.2 (Jönsson \& Eklundh 2004). Las estimaciones fueron realizadas sobre 2000 puntos seleccionados aleatoriamente dentro de cada PA. Este método se basa en el cálculo de una regresión polinomial local (de grado k), con al menos $\mathrm{k}+1$ puntos equiespaciados, para determinar el nuevo valor de cada punto (Savitsky \& Golay 1964). El resultado es una función similar a los datos de entrada, pero suavizada. La principalventaja de esta aproximación es que tiende a preservar características de la distribución inicial tales como los máximos y 
mínimos relativos, así como el ancho de los picos, que normalmente desaparecen con otras técnicas de promediado (como la media desplazada). Sobre estas curvas promedio se identificaron el número de estaciones de crecimiento y se derivaron los parámetros biométricos a) tasa de incremento del IVN, b) tasa de caída del IVN c) inicio de estación de crecimiento, d) momento de máximo IVN, e) fin de estación de crecimiento, f) valor máximo IVN, g) valor basal de IVN, h) IVN acumulado anual. Se consideró como inicio de estación de crecimiento cuando el IVN logró un incremento sostenido de $20 \%$ superior al IVN mínimo. Por oposición, el fin de la estación de crecimiento fue definido cuando el IVN disminuyó de manera sostenida y alcanzó un valor $20 \%$ superior al IVN mínimo.

\section{Re-escalado del mapa de suelos}

Para asociar los PA al tipo de suelo se mejoró la resolución espacial del mapa de suelos del INTA (1977) en la zona de estudio a partir de la ecuación suelo-paisaje propuesta por Dokuchaev (1886). Se incorporó a la información existente información paisajística detallada derivada de sensores remotos. Los modelos digitales de terreno disponibles, como el SRTM 90 m (NASA-NGA 2000) o el ASTER GDEM2 30 m (Tachikawa et al. 2011), poseen una resolución altimétrica insuficiente $(\geq 1 \mathrm{~m})$ para la identificación de cubetas y microdepresiones, características del relieve en "mosaico" de la región (Miaczynski 1995). Vázquez et al. (2003) obtuvieron un elevado coeficiente de correlación entre el mapa de frecuencias de anegamiento de la cuenca del Azul y los suelos dominantes en las unidades cartográficas del mapa de suelos 1: 50000. Para "revelar" el paisaje en zonas anegables, se utilizó el mapa de riesgo hídrico de la Pampa Deprimida escala 1:25000 desarrollado por Vázquez et al. (2009). Para las áreas planas no anegables, lomas y serranías, se utilizó el MDT SRTM (NASA-NGA 2000), corregido para el área de estudio por Masuelli et al. (2006). La asignación de subgrupos de suelos y valores de propiedades

Tabla 1. Superficie ocupada por los PA identificados en la Pampa Deprimida.

Table 1. Area occupied by PA identified in the flooding Pampas.

\begin{tabular}{ccc}
\hline PA & Superficie (ha) & $\%$ \\
\hline 1 & 465383 & 9 \\
2 & 227492 & 4 \\
3 & 1094080 & 21 \\
4 & 1317001 & 25 \\
5 & 330020 & 6 \\
6 & 108705 & 2 \\
7 & 219156 & 4 \\
8 & 69092 & 1 \\
9 & 385753 & 7 \\
10 & 343768 & 7 \\
11 & 483641 & 9 \\
12 & 94533 & 2 \\
Total & 5194673 & \\
\hline
\end{tabular}

edáficas al paisaje se realizó considerando las relaciones suelo-paisaje descritas en las unidades cartográficas del mapa de suelos 1:50000 (INTA 1977-1989). Para la validación, se realizó un análisis de correspondencia entre los datos modelados y 244 perfiles relevados en 12 establecimientos ubicados en zonas con características edáficas contrastantes y zonas de transición.

\section{Asociación entre PA y comunidades vegetales}

Mediante un análisis de correspondencia se compararon los PA y 8259 identificaciones de comunidades vegetales geoposicionadas, observadas en una transecta de $2000 \mathrm{~km}$ realizada en sectores ambientalmente representativos. Esto permitirá evaluar la capacidad del algoritmo KMEANS para discriminar comunidades vegetales herbáceas destinadas al aprovechamiento ganadero, como son los pastizales y las pasturas cultivadas o naturalizadas.

\section{Asociación entre PA y suelos}

La relación existente entre las comunidades vegetales y el suelo ha sido estudiada ampliamente en la región (Deregibus \& Cauhépé 1983; Burkart et al. 1990; Burkart 1998; Perelman et al. 2001); se delimitaron ambientes característicos para cada una de ellas y se generaron mapas de distribución potencial (Burkart et al. 2005). Conociendo la capacidad de adaptación de las comunidades vegetales a un rango de condiciones edáficas se realizó un análisis discriminante con procedimiento stepwise ( $\alpha$ ingreso $=0.1, \alpha$ salida $=0.15$ ) para identificar la relación existente entre los PA y las propiedades del suelo extraídas del mapa de suelos ajustado. Las propiedades seleccionadas fueron a) espesor del epipedón (Esp_epip), b) Ph del epipedón (Ph_epip), c) porcentaje de sodio de intercambio del epipedón (PSI_epip), d) agua útil del epipedón (AU_epip), e) profundidad del horizonte B argílico (Prof_Bar), f) porcentaje de sodio de intercambio del horizonte B argílico (PSI_Bar), g) conductividad eléctrica de la sección de control (CE_sc), h) profundidad donde se observan condiciones ácuicas (Acuic) e i) profundidad de tosca (Tosca). El análisis se realizó sobre una muestra aleatoria de 8000 puntos extraída de toda la región (SYSTAT 11, (CSYSTAT Soft 2004).

\section{Resultados}

\section{Identificación de PA}

Se identificaron 12 PA de pastizales en la Pampa Deprimida que cubrían una superficie de 5190000 ha (74\% del área total) (Tabla 1). Este valor es levemente inferior a las 17 comunidades descritas por Verboorst (1967). Cuatro de ellos representan el 62\% de la superficie ocupada por pastizales en la Pampa Deprimida (3, 4, 9 y 11), y sólo dos 


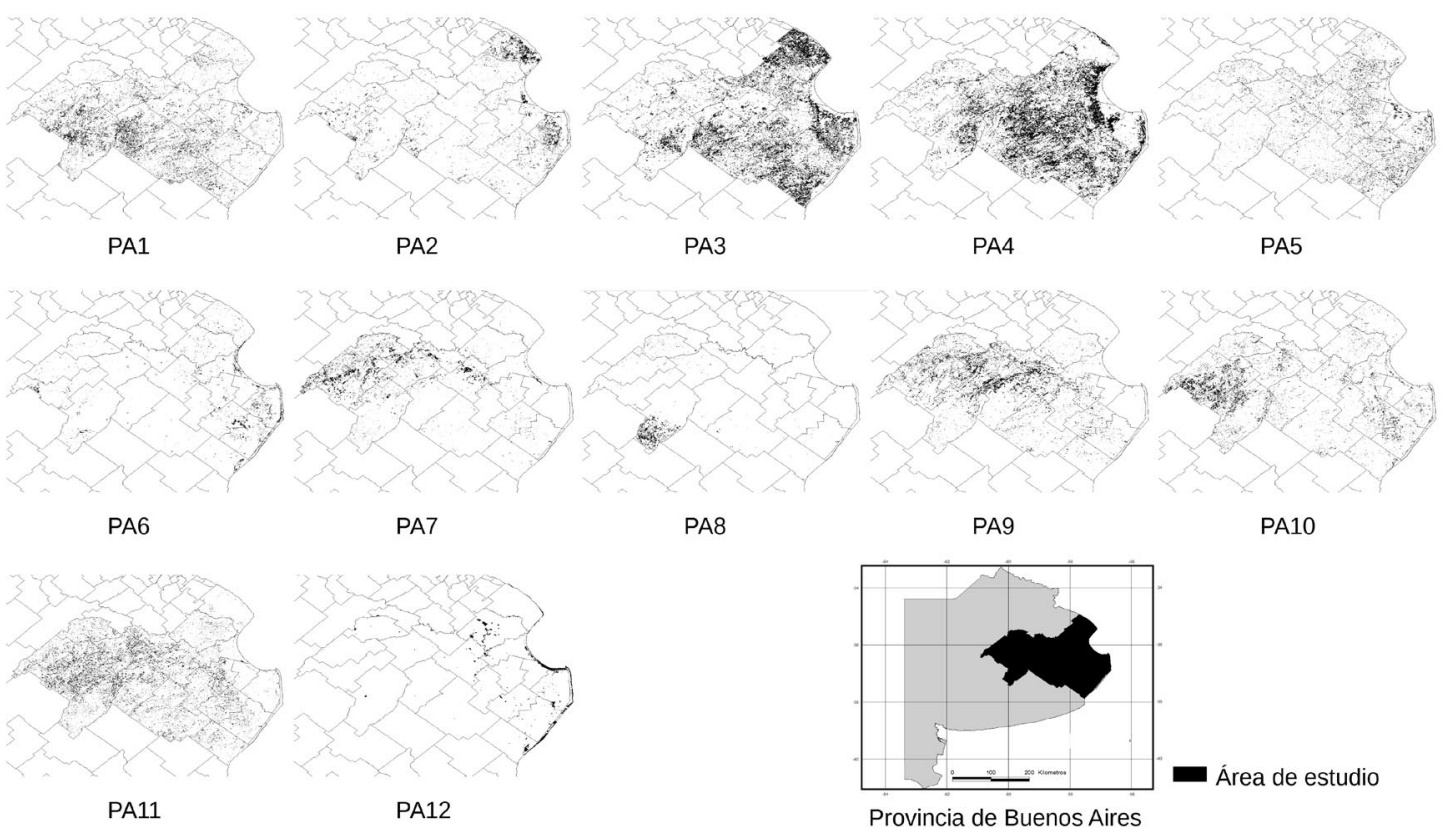

Figura 1. Distribución de los PA identificados en la Pampa Deprimida.

Figure 1. PA distribution over the flooding Pampas.

de ellas (3 y 4) cubren 2400000 ha, lo cual las convierte en los PA más representativos de la región (Figura 1).

Los PA fueron agrupados según el número de estaciones de crecimiento y la amplitud detectada en el IVN (Figura 3). Los PA 1, 5, 6 y 12 presentan curvas del tipo unimodal, con una amplitud del IVN $\leq 0.15$. Los PA $7,9,10$ y 11 también presentan curvas del tipo unimodal, pero con una amplitud del
IVN $\geq 0.2$. Finalmente, los PA 2, 3, 4 y 8 se caracterizaron por presentar una curva anual bimodal (Figura 2, Tabla 2).

El mes de inicio de estación de crecimiento es principalmenteagosto. LosPA 2,6y 7, ubicados en las zonas de la costa y de transición con el oeste arenoso, comenzaron en septiembre. El final del ciclo de crecimiento primaveral se presentó en los meses de diciembre y enero, asociados a la región central y las de transición

Tabla 2. Biometría de los PA identificados en la Pampa Deprimida ( $L$ der=tasa de incremento IVN, Rder=tasa de caída IVN,$i=$ inicio de estación de crecimiento, $p=$ momento de IVN máximo, $f=$ final de estación de crecimiento, $I V N$ máx $=$ valor de IVNmáximo, $I V N$ base= valor de IVN mínimo, IVN=integral anual de IVN).

Table 2. Biometry of the PA identified in the flooding Pampas (Lder=IVN increment rate, Rder=IVN falling rate, $i=$ start of growing season, $p=$ time of maximum IVN, $f=$ end of growing season, $I V N$ máx.=maximum IVN value, IVN base=minimum IVN value, IVN= annual IVN integral).

\begin{tabular}{|c|c|c|c|c|c|c|c|c|c|c|c|c|c|c|}
\hline \multirow[t]{3}{*}{$\mathrm{PA}$} & \multicolumn{6}{|c|}{ Crecimiento primavera } & \multicolumn{6}{|c|}{ Crecimiento verano } & \multirow{3}{*}{$\begin{array}{c}\text { IVN base } \\
\pm \text { desvío estándar }\end{array}$} & \multirow{3}{*}{$\int \mathrm{IVN}$} \\
\hline & \multicolumn{3}{|c|}{ Tasa evolución i } & \multirow{2}{*}{$\begin{array}{c}\mathrm{p} \\
\text { mes }\end{array}$} & \multirow[t]{2}{*}{$\mathrm{f}$} & \multirow[t]{2}{*}{$\begin{array}{c}\text { IVN máx. } \\
\pm \text { desvío estándar }\end{array}$} & \multicolumn{2}{|c|}{$\begin{array}{l}\text { Tasa evolución } \\
\text { IVN mensual }\end{array}$} & \multirow[t]{2}{*}{ i } & \multirow{2}{*}{$\begin{array}{c}\mathrm{p} \\
\mathrm{mes}\end{array}$} & $\mathrm{f}$ & \multirow[t]{2}{*}{$\begin{array}{l}\text { IVN } \\
\text { máx. }\end{array}$} & & \\
\hline & Lder & Rder & $\mathrm{i}$ & & & & Lder & Rder & & & & & & \\
\hline 1 & 0.071 & -0.027 & 8 & 10 & 1 & $0.72 \pm 0.020$ & 0.036 & -0.059 & 2 & 3 & 6 & 0.73 & $0.53 \pm 0.016$ & 7.63 \\
\hline 2 & 0.044 & -0.029 & 9 & 10 & 12 & $0.66 \pm 0.031$ & 0.033 & -0.036 & 2 & 3 & 6 & 0.66 & $0.56 \pm 0.026$ & 7.34 \\
\hline 3 & 0.053 & -0.040 & 8 & $10-11$ & 1 & $0.74 \pm 0.023$ & 0.038 & -0.043 & 2 & 3 & 6 & 0.72 & 022 & 7.98 \\
\hline 4 & 0.076 & -0.019 & 8 & $10-11$ & 12 & 0.74 & 0.021 & -0.072 & 2 & 3 & 6 & 0.75 & 0.5 & 7.95 \\
\hline 5 & 0.054 & -0.040 & 8 & 10 & 1 & $0.68 \pm 0.024$ & 0.014 & -0.059 & 2 & 3 & 6 & 0.72 & 023 & 7.69 \\
\hline 6 & 0.051 & -0.039 & 9 & 10 & 12 & 0.5 & 0.020 & -0.045 & 1 & 3 & 6 & 0.62 & 0. & 6.56 \\
\hline 7 & 0.063 & -0.080 & 9 & $11-3$ & 6 & $0.66 \pm 0.033$ & 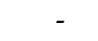 & 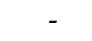 & - & - & - & - & $0.42 \pm 0.025$ & 6.91 \\
\hline 8 & 0.084 & -0.048 & 7 & 10 & 12 & $0.65 \pm 0.064$ & 0.110 & -0.110 & 1 & 2 & 6 & 0.74 & $0.38 \pm 0.042$ & 6.66 \\
\hline 9 & 0.069 & -0.084 & 8 & $11-3$ & 6 & $0.70 \pm$ & - & - & - & - & - & - & $0.54 \pm 0.023$ & 7.31 \\
\hline 10 & 0.066 & -0.059 & 8 & $10-3$ & 6 & $0.65 \pm 0.023$ & 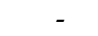 & - & - & - & - & - & $0.52 \pm 0.026$ & 7.11 \\
\hline 11 & 0.075 & -0.069 & 8 & $10-3$ & 6 & $0.69 \pm 0.024$ & - & - & - & - & - & - & $0.58 \pm 0.021$ & 7.42 \\
\hline 12 & 0.082 & -0.070 & 9 & 10 & 12 & $0.18 \pm 0.180$ & 0.014 & -0.018 & 1 & $2-3$ & 8 & 0.22 & $0.14 \pm 0.130$ & 2.08 \\
\hline
\end{tabular}



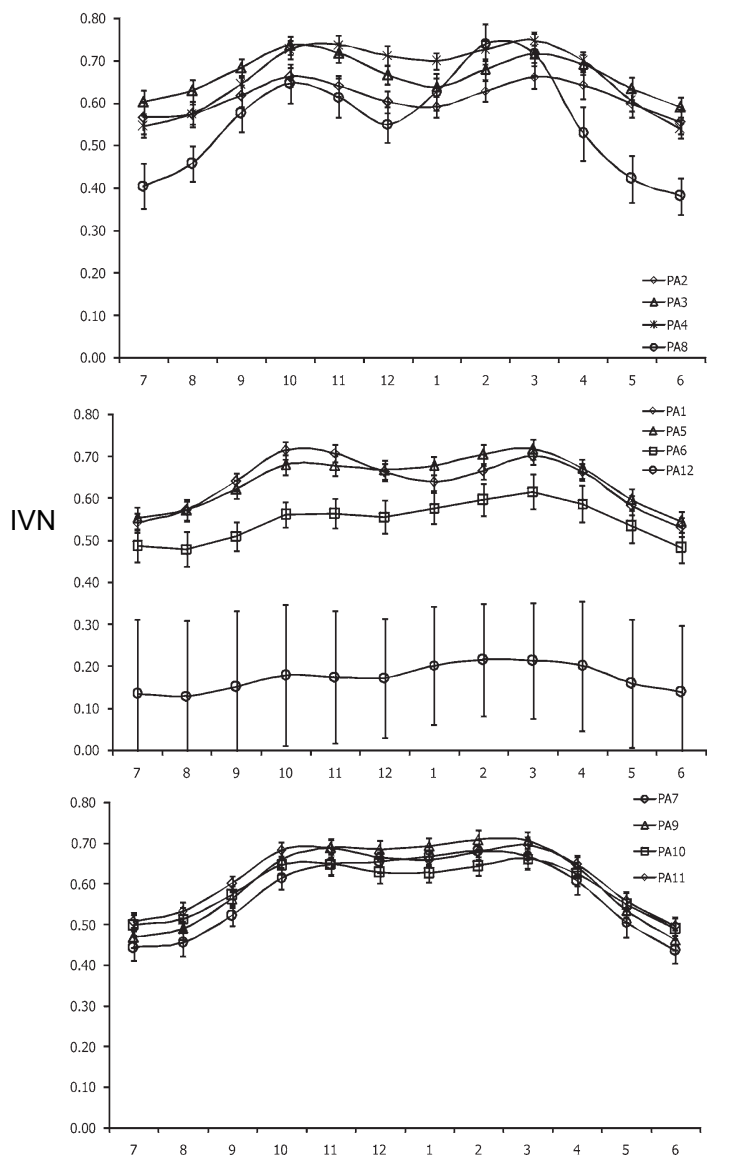

El crecimiento de verano se caracteriza por poseer tasas de evolución del IVN inferiores y valores máximos de IVN mayores a los hallados en primavera. Esto responde a que las altas tasas de crecimiento de primavera generan rápidamente un excedente de material muerto y una senescencia por floración que afectan la respuesta espectral en primavera. Durante el verano y otoño, predomina el crecimiento vegetativo con menores tasas de crecimiento, y por ende, menor acumulación de material muerto. Los valores base de IVN de la Pampa Deprimida son en general elevados (mayores a 0.5) lo cual indica que a esta escala de estudio no habría importantes áreas con suelo desnudo o sin actividad vegetal prolongada. El IVN acumulado anual presenta grupos bien delimitados, con valores en los rangos menor a 5, 6.0-6.5, 6.5-7.0, 7.0-7.5 y > 7.5. El primero, por su distribución, se asocia con claridad a la presencia dominante de especies palustres o comunidades D puras, mientras que el último evidenciaría una dominancia de especies implantadas o naturalizadas (principalmente Festuca sp.), como se observó durante la ejecución del muestreo.

Mapa de suelos detallado de la cuenca del Salado

A partir del mapa de suelos del INTA 1: 50000 (1977-1989) se identificaron 173 áreas con secuencias de paisaje-suelo homogéneas. Con ayuda del MDT y del mapa de riesgo hídrico, se delimitaron las áreas ocupadas por cada subgrupo de suelos dentro de la Pampa Deprimida. Se verificó a campo el alto grado de correlación que existe entre los

con la pampa ondulada y sistema de Tandilia, respectivamente (PA 3 y 4 ).

Tabla 3. Matriz de confusión entrelos subgrupos de suelos (SGS) observados y predichos para la Pampa Deprimida a partir del modelo digital de terreno y el mapa de riesgo hídrico $(n=244)$ (Udips=Udipsament, Arg.t=Argiudol típico, Hta=Hapludol tapto árgico, Htn=Hapludol tapto nátrico, Ntrd=Natrudol, Nol=Natracuol típico, Nalf=Natracualf típico, Ver=Peludertes nátricos o sálicos, Acuol=Argiacuol, Lag=Laguna).

Table 3. Confusion matrix between observed and predicted soil subgroups (SGS)for the flooding Pampas using the digital terrain model and the flooding risk map $(n=244)$. (Udips=Udipsament, Arg.t=tipic Argiudol, Hta=tapto argic Hapludol, Htn=tapto natric Hapludol, Ntrd=Natrudol, Nol=tipic Natracuol, Nalf=tipic Natracualf, Ver=natric or salic Peludert, Acuol=Argiacuol, Lag=Lakes or ponds).

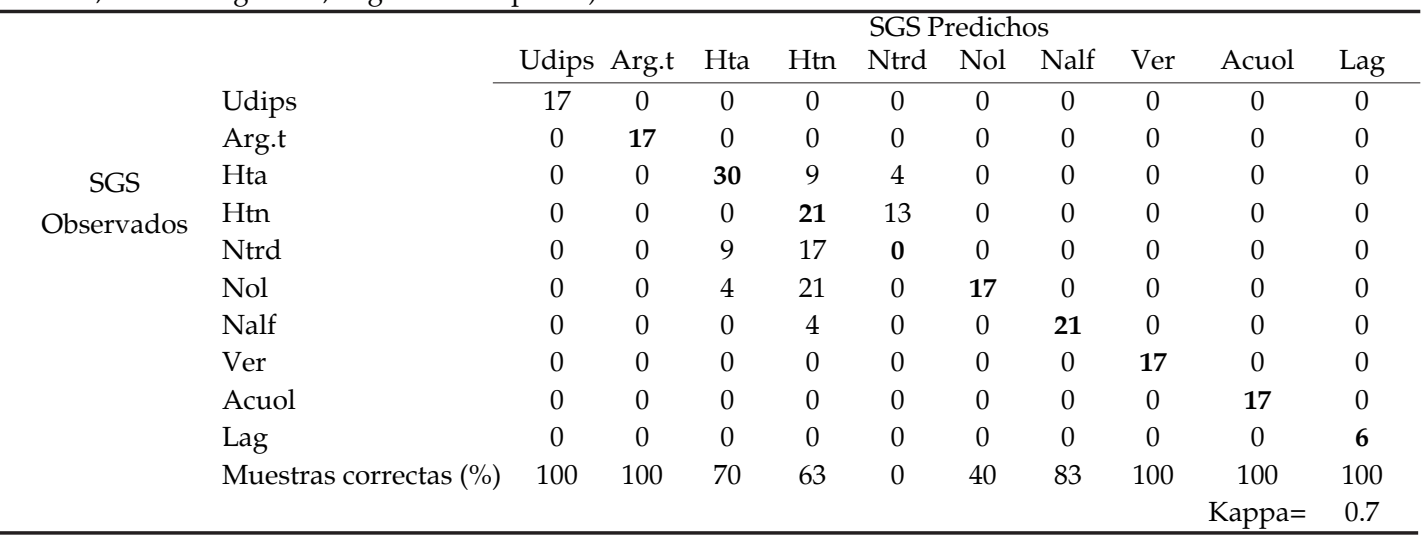




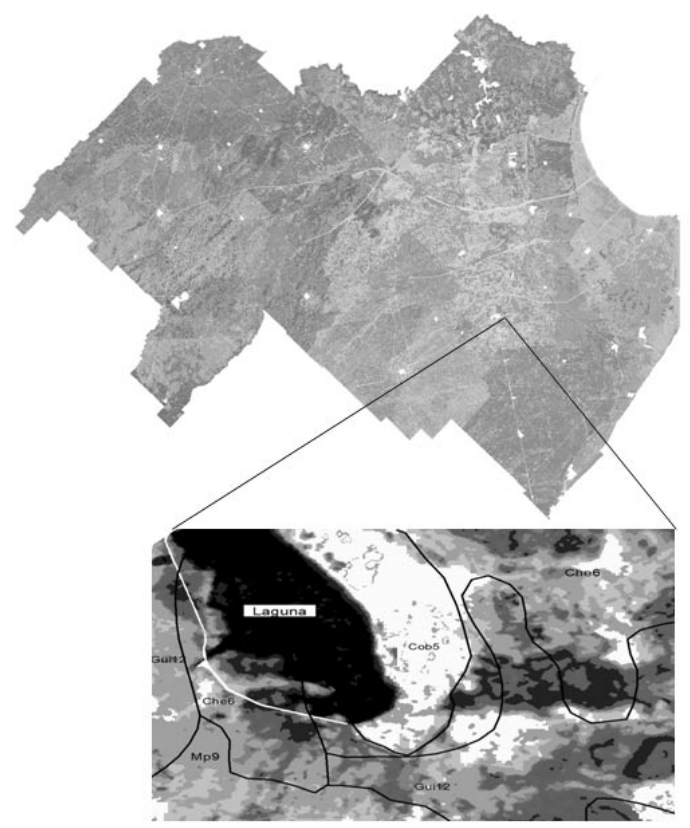

Figura 3. Cartografía edáfica 1:25.000 resultante para la Pampa Deprimida. Las líneas delimitan áreas de complejos de suelo identificados a escala 1:50000, los tonos de gris identifican la ubicación de los subgrupos de suelos.

Figure 3. Resultant 1:25000 edaphic cartography for the flooding Pampas. Lines limit 1:50000 soil subgroup complexes, greys tones identify soil subgroups ubication.

suelos con deficiente drenaje y problemas de sodicidad respecto del mapa de riesgo hídrico, y entre los suelos bien drenados y el modelo de relieves. Se obtuvo un kappa de 0.7 contrastando los suelos modelados a nivel de subgrupo de suelos, según el sistema de Taxonomía de Suelos (Soil Survey Staff 2010), con las verdades de campo (Tabla 3). Cuando se reagruparon los subgrupos de suelo contemplando el tipo de limitante, el kappa fue de 0.9 (Tabla 4).

Estos resultados concuerdan conlosobtenidos a partir de encuestas realizadas en más de 1000 establecimientos que recibieron el mapa de suelos detallado en su etapa preliminar, lo cual permite validar este producto a escala 1 : 25000 como versión final (Figura 3).

\section{Asociación entre PA y comunidades vegetales}

Se encontró una asociación fuerte entre los PA y las comunidades vegetales (kappa $=0.86$ ). Si bien el pastizal está típicamente constituido por un mosaico de comunidades de algunas decenas de $\mathrm{m}^{2}$, la evolución del IVN medio de cada píxel resumiría la participación relativa de las distintas comunidades dentro de la unidad de muestreo. En términos generales, cada PA representa a una o dos comunidades dominantes, ocupando combinadas más de $70 \%$ de la superficie de cada caso (Tabla 5). Varios PA representan comunidades vegetales casi puras, como PA 1 (Cynodon sp.), PA 2 (D), PA 8 (serranas), PA 9 (C) y PA 12 (palustres). Cuando se analiza el grado de confusión desde el punto de vista de la comunidad vegetal (proporción de cada comunidad

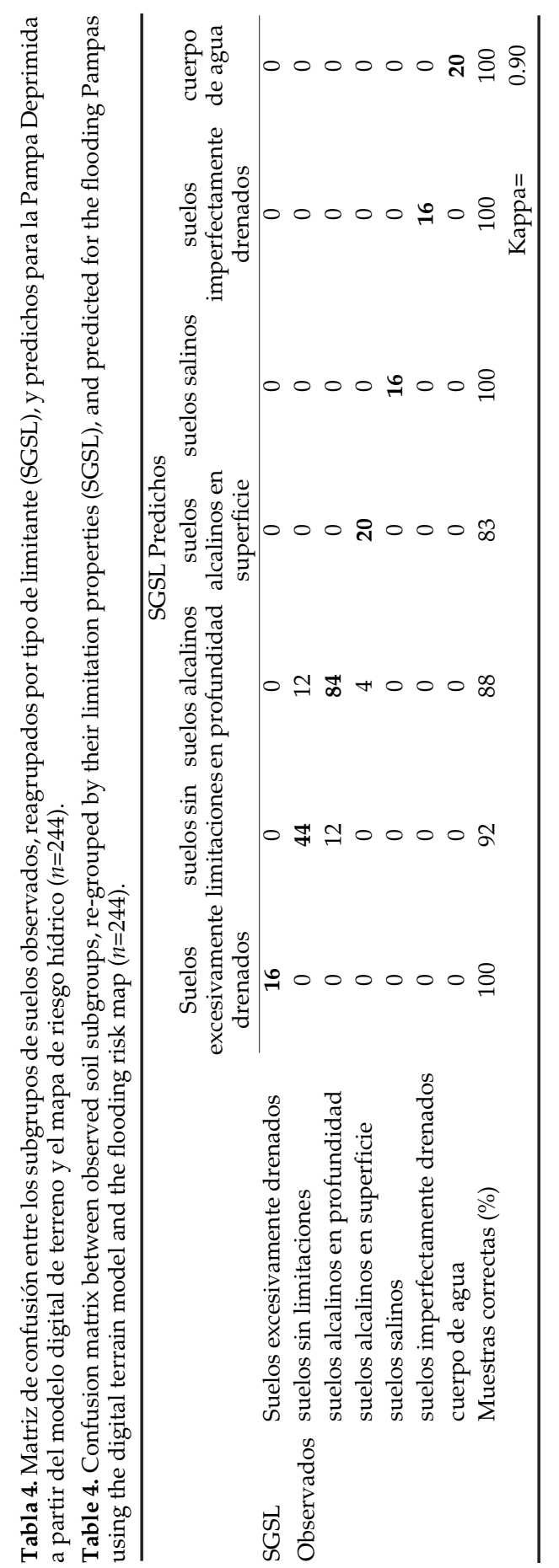


Tabla 5. Matriz de confusión entrecomunidades vegetales identificadas durante las observaciones realizadas en la Pampa Deprimida y los PA identificados $(n=8259)$.

Table 5. Confusion matrix between vegetal communities identified during observations realized in the flooding Pampas and the identified PA $(n=8259)$.

\begin{tabular}{|c|c|c|c|c|c|c|c|c|c|c|c|c|c|}
\hline \multirow[b]{2}{*}{ Comunidades } & \multicolumn{12}{|c|}{ PA } & \multirow[b]{2}{*}{ Certeza \% } \\
\hline & 1 & 2 & 3 & 4 & 5 & 6 & 7 & 8 & 9 & 10 & 11 & 12 & \\
\hline Cynodon sp. & 2524 & 18 & 4 & & & & & & 13 & & & & 0.99 \\
\hline $\mathrm{D}$ & 45 & 86 & 72 & & & & & & & & 10 & & 0.40 \\
\hline $\mathrm{B}+$ Estipa sp. & 11 & & 167 & & & & & & & & 17 & & 0.86 \\
\hline B4+Festuca sp.+Raygrass sp. & 4 & & 214 & 3272 & & & & & & & & & 0.94 \\
\hline $\mathrm{B}+\mathrm{C}+\mathrm{D}+\mathrm{PALUSTRE}$ & & & & & 29 & & & & & & & & 1.00 \\
\hline Festuca sp.+B & & & 92 & 138 & & 45 & & & 121 & & & & 0.11 \\
\hline Pajonal+C & & & & & & & 15 & & & & & & 1.00 \\
\hline SERRANA & & & & & & & & 19 & & & & & 1.00 \\
\hline $\mathrm{C}$ & & & 14 & 14 & & & & & 490 & & & & 0.95 \\
\hline Agropiron sp. + Festuca sp.+C & & & & & & & & & & 451 & 16 & & 0.97 \\
\hline PAJONAL & 73 & & 3 & 173 & & & & & 20 & 3 & 35 & & 0.11 \\
\hline PALUSTRE & 7 & 26 & & & & & & & & 8 & & 10 & 0.20 \\
\hline Certeza \% & 0.95 & 0.66 & 0.30 & 0.91 & 1.00 & 1.00 & 1.00 & 1.00 & 0.76 & 0.98 & 0.45 & 1.00 & \\
\hline
\end{tabular}

o combinación de ellas representada por cada PA), las de mayor confusión son las comunidades D, Festuca sp.+B, pajonal y palustres. Si analizamos la especificidad de los PA para identificar combinaciones únicas de comunidades vegetales los de mayor confusión son los PA 3 y 11. Es destacable la superficie ocupada por Festuca sp.; es el componente botánico de mayor importancia en los PA 3 y 4 , los que ocupan $50 \%$ de la superficie total estudiada, y participa como componente secundario en otros PA (Tabla 5). Esto indica una dominancia de la especie cultivada Festuca sp., la cual desplazó en importancia a las comunidades autóctonas.
Un análisis detallado de los PA 3 y 4 determinó que a pesar de tener la misma curva estacional de IVN, la región al este de la Ruta Nacional 2 se caracterizó por la presencia casi exclusiva de las comunidades B, B4 y Raygrass sp., mientras que en el resto dominó Festuca sp.

\section{Asociación entre PA y propiedades del suelo}

Todas las propiedades del suelo fueron retenidas por el modelo, siendo PSI_Bar y Esp_ epip las más asociada a los PA (Wilk's $?=0.26$ y 0.27) (Tabla 6). El análisis discriminante demostró que existe una correlación entre los PA y las propiedades del suelo (Kappa=0.65).

Tabla 6. Propiedades edáficas medias estimadas a partir de un análisis discriminante para los 12 PA identificados en la Pampa Deprimida: espesor del epipedón (Esp_epip), Ph del epipedón (Ph_epip), porcentaje de sodio de intercambio del epipedón (PSI_epip), agua útil del epipedón (AU_epip), profundidad del horizonte B argílico (Prof_Bar), porcentaje de sodio de intercambio del horizonte B argílico (PSI_Bar), conductividad eléctrica de la sección de control (CE_sc), profundidad donde se observan condiciones ácuicas (Acuic) y profundidad de tosca (Tosca) $(n=8000)$.

Table 6. Average soil properties estimated by a discriminant analysis for the 12 PA identified in the flooding Pampas: epipedón thickness (Esp_epip), epipedon Ph (Ph_epip), epipedón exchangeable sodium percentage (PSI_epip), epipedón available water (AU_epip), argilic B horizon depth (Prof_Bar), exchangeable sodium percentage of the argilic B horizon (PSI_Bar), electric conductivity of the control section (CE_sc), acuic conditions depth (Acuic) andpetrocalcic horizon depth (Tosca) $(n=8000)$.

\begin{tabular}{lccccccccccccc}
\hline & \multicolumn{10}{c}{ PA } & \multicolumn{10}{c}{ Wilk's $\lambda$} \\
\cline { 2 - 12 } Propiedades edáficas medias & 1 & 2 & 3 & 4 & 5 & 6 & 7 & 8 & 9 & 10 & 11 & 12 & Wilk \\
PSI_Bar (s/u) & 29 & 32 & 24 & 25 & 32 & 17 & 18 & 1 & 28 & 20 & 26 & 0 & 0.26 \\
Esp_epip (cm) & 25 & 17 & 22 & 18 & 19 & 40 & 41 & 30 & 26 & 30 & 34 & 0 & 0.27 \\
AU_epip (mm) & 49 & 37 & 36 & 36 & 38 & 68 & 46 & 63 & 43 & 50 & 67 & 0 & 0.28 \\
CE_sc (dS/m) & 0 & 2 & 2 & 3 & 2 & 0 & 0 & 0 & 2 & 1 & 1 & 0 & 0.28 \\
PSI_epip (s/u) & 23 & 16 & 8 & 7 & 19 & 7 & 12 & 1 & 31 & 11 & 19 & 0 & 0.29 \\
Prof_Bar (cm) & 27 & 54 & 63 & 70 & 42 & 42 & 57 & 28 & 34 & 38 & 32 & 0 & 0.29 \\
Acuic (cm) & 68 & 29 & 54 & 26 & 25 & 42 & 90 & 500 & 35 & 32 & 50 & 0 & 0.31 \\
Tosca (cm) & 111 & 188 & 177 & 190 & 180 & 141 & 183 & 32 & 182 & 139 & 155 & 0 & 0.31 \\
Ph_epip (s/u) & 7 & 8 & 6 & 6 & 7 & 6 & 7 & 7 & 6 & 6 & 6 & 0 & 0.32 \\
N & 2662 & 125 & 469 & 3566 & 28 & 45 & 15 & 41 & 644 & 454 & 78 & 10 & \\
\hline
\end{tabular}


Tabla 7. Matriz de confusión entre PA observados y predichos utilizando las variables edáficas modeladas a escala detallada $(n=8000)$.

Table 7. Confusion matrix between observed and predicted PA using modeled soil properties at detailed scale $(n=8000)$.

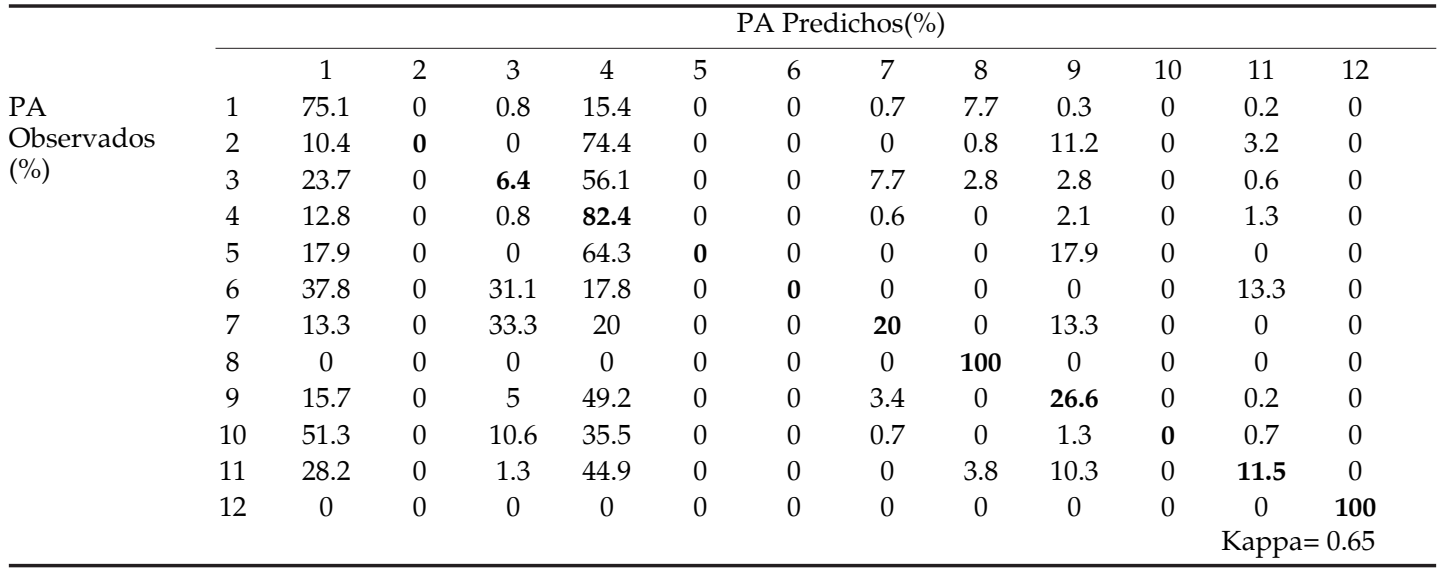

Los PA 1, 4, 8 y 12 presentaron una asociación específica a determinadas propiedades del suelo, por lo cual pudieron ser predichos casi en su totalidad (Tabla 7). Los PA 2, 5, 6 y 10 no pudieron ser predichos por el modelo, mientras que el resto presentó variados niveles de confusión.

\section{DisCUSIÓN}

La distribución de los PA y las formas que presentaron las curvas de evolución medias de IVN son indicadores de comportamientos diferenciales de la cubierta vegetal herbácea de la Pampa Deprimida a pesar de que la unidad de estudio (píxel=6.25 ha) es lo suficientemente amplia como para absorber gran parte de la variabilidad espacial edáfica y, por ende, de las comunidades. Al comparar la biometría de estos PA se infiere que su composición incluyó más de una comunidad. Las curvas unimodales solo representan el solapamiento de varias curvas de evolución de IVN pertenecientes a las diferentes comunidades vinculadas espacialmente.

La ecuación suelo-paisaje propuesta por Dokuchaev (1886), complementada con información derivada a partir de sensores remotos, funcionó de forma correcta. El MDT no presentó el suficiente detalle para detectar variaciones del paisaje en zonas de llanura. La incorporación de la frecuencia de anegamiento a la ecuación suelo-paisaje fue definitoria. Existen MDT de mayor resolución espacial, como el SRTM de $30 \mathrm{~m}$, y de mayor resolución altimétrica, como los productos LIDAR (Light Detection and Ranging). El primero es el producto del que derivó el SRTM de $90 \mathrm{~m}$ utilizado en este trabajo (por re-escalado de sus píxeles). Su versión original es "ruidosa" ya que es sensible a pequeñas variaciones de la superficie, como la presencia de árboles, y no presenta mejor resolución altimétrica. El segundo producto presenta una resolución altimétrica del orden de los $15 \mathrm{~cm}$. Puede identificar a partir de un post procesamiento la altura del dosel vegetal y separarlo de la altimetría del terreno. Su mayor desventaja es su elevado costo ya que son transportados por aviones. Son ideales para relevamientos de pequeñas áreas. Esto hizo del MDT de $90 \mathrm{~m}$ una opción ideal para trabajar grandes regiones. La sensibilidad escasa del MDT pudo ser complementada de manera satisfactoria al aplicar el concepto "de equilibrio hidrostático", en el cual el agua ocupa los sitios de menor energía cinética. Así se detectaron las áreas bajas del terreno y su profundidad inferida a partir de su frecuencia de anegamiento. Los resultados fueron consistentes con los mencionados por otros autores (Burrough et al. 2000; Zhu et al. 2001). Se corroboró la exactitud del mapeo de suelos para subgrupos de suelo y para propiedades edáficas de diagnóstico, lo que permitió validar este producto como una versión final (Tabla 3). El mapa de suelos de la Pampa Deprimida escala 1:25000 está disponible para su uso en [http: //geointa.inta.gov.ar/node/11id\%D23].

Si bien la actividad predominante en Pampa Deprimida sigue siendo la ganadera, en los últimos 10 años la actividad agrícola se incrementó en 300\% y desplazó la ganadería hacia sitios más marginales, sin disminución del número de cabezas (Vázquez et al. 2008). Estos aspectos podrían haber modificado la 
distribución, composición y comportamiento de los pastizales de la región. Durante la realización del muestreo, se evidenció que Festuca sp. ocupa la mayor parte de la región, desplazando a las especies nativas del sistema. Lo mismo sucedió con el agropiro (Agropiron sp.), principalmente en la zona con tosca del oeste (PA 10 y 11). Estas especies cultivadas fueron introducidas en la región durante el período 1969-1979 por el Plan Balcarce (International Bank for Reconstruction and Development 1967) naturalizándose de manera muy exitosa. Por lo tanto, sería necesario revisar hasta qué punto debemos seguir definiendo de manera generalizada a la Pampa Deprimida como un área cubierta por pastizales naturales. Quizás sería más próximo a la realidad definirla como un área ocupada por pasturas implantadas, naturalizas y bajo diferentes condiciones de degradación de Festuca sp., con relictos del pastizal natural originario en determinados sectores. Las áreas ocupadas por Cynodon sp. (PA 1) coinciden con sitios utilizados de forma esporádica para agricultura durante los últimos 15-20 años y que luego fueron abandonados.

La asociación existente entre las comunidades vegetales y el rango de suelos que ocupan dentro de determinados paisajes, ha sido señalada repetidas veces en las descripciones de la vegetación (Vervoorst 1967; León et al. 1979; Batista et al. 1988; Burkart et al. 1990, 1998). Sucedió lo mismo cuando se buscó esta correspondencia considerando la evolución temporal del IVN. Las cubiertas vegetales se adaptan a un gradiente de condiciones ambientales, excepto en situaciones extremas. Por lo tanto, un pixel puede incluir varias comunidades vegetales que coexisten sobre dos o tres tipos de suelo sin que éstos afecten de forma significativa su comportamiento espectral, pero estarán subordinadas al grado delimitación queleimpondránlas propiedades edáficas. Como ejemplo, los PA más asociados a las propiedades del suelo fueron los ubicados sobre áreas afectadas por un fuerte disturbio, como sitios cultivados y abandonados (PA 1 ), áreas con problemas de salinidad (PA 4), sierras (PA 8) o ambientes lacustres (PA 12). Estos resultados mantienen coherencia con los resultados modelados por Burkart et al. (2005). Los PA 2, 5, 6 y 10 fueron reasignados a otros PA con similares propiedades edáficas pues no presentaron ninguna en particular que permita discriminarlos del resto. Los PA 2 y 5 (10\% de la superficie total) fueron predichos como PA 4, principalmente por similitudes en el porcentaje de sodio de intercambio del horizonte B argílico, el espesor del epipedón y la profundidad de la tosca. Los PA 6 y 10 ( $9 \%$ de la superficie total) fueron predichos principalmente como PA 1, pues comparten similitudes en el espesor del epipedón y la profundidad de la tosca. Si bien hubo confusión desde el punto de la clasificación, los PA 2, 5, 6 y 10 podrían desarrollarse bajo las condiciones predichas.

\section{CONCLUSIONES}

El análisis de la serie temporal anual media de IVN obtenida por el sensor MODIS permitió discriminar 12 PA de pastizales y pasturas, estimar su biometría y confirmar su asociación con las comunidades vegetales y a las propiedades edáficas en la Pampa Deprimida.

La asociación entre los PA y las propiedades edáficas fue muy específica bajo condiciones limitantes para el crecimiento vegetal, observable en suelos muy someros, salinos o inundados gran parte del año. También lo fue en áreas afectadas por procesos de agriculturización y luego abandonadas. Las comunidades vegetales tienen la capacidad de adaptarse a situaciones edáficas diferentes, atenuando el efecto suelo a la escala de trabajo aplicada y en coincidencia con lo expresado por varios autores.

El desplazamiento de especies nativas por las cultivadas (principalmente Festuca sp.) es notable en gran parte de la Pampa Deprimida. El único sector que aún preserva un estado conservado es el costero, que por su marginalidad productiva a quedado relativamente "protegido" de la antropización.

\section{BiBLIOGRAFÍA}

Allen, T; R O'NeILl \& S HoEKSTRA 1984. Interlevel relations in ecological research and management: some working principles from hierarchical theory. Rocky Mountain Forest and Range Experiment Station. Thechnical report RM-110, Fort Collins, Co.

Batista, W; M Taboada; R Lavado; S Perelman \& R León. 2005. Asociación entre comunidades vegetales y suelos en el pastizal de la Pampa Deprimida. Pp. 113-129 en: Oesterheld, M; M Aguiar; C Ghersa \& JM Paruelo (eds.). La heterogeneidad de la vegetación de los agroecosistemas. Editorial Facultad de Agronomía.

BAtista, W; R León \& S Perelman. 1988. Las comunidades vegetales de un pastizal natural de la región de Laprida, prov. de Buenos Aires, Argentina. Phytocoenologia, 16:465-480.

Berasastegui, L \& L Barberis. 1982. Los suelos de las comunidades de Castelli - Pila, depresión del Salado, 
Provincia de Buenos Aires. Revista de la Facultad de Agronomía, 3:13-25.

Bocchetтo, C. 1979. Marco conceptual para caracterizar sistemas reales de producción agropecuaria, asociado al proceso de cambio tecnológico. INTA, EEA Balcarce. Pp. 43.

Boles, S; X XIAO; J Liu; Q Zhang; S MunKhtuYa; ET AL. 2004. Land cover characterization of Temperate East Asia using multi-temporal VEGETATION sensor data. Remote Sensing of Environment, 90:477-489.

Burkart, S; R León; S Perelman \& M Agnusdei. 1998. The grasslands of the flooding Pampa (Argentina): floristic heterogeneity of natural communities of the southern río Salado basin. Coenoses, 13:17-27.

BURKART, S; M GARBULSKY; C GHERSA; J GUERSCHMAN; R LeÓN; ET AL. (ex aequo). 2005. Las Comunidades Potenciales del Pastizal Pampeano Bonaerense. Pp. 379-399 en: Oesterheld, M; M Aguiar; C Ghersa \& JM Paruelo (eds.). La heterogeneidad de la vegetación de los agroecosistemas. Un homenaje a Rolando León. Editorial Facultad de Agronomía, UBA. Buenos Aires, Argentina.

BurKart, S; R León \& C Movia. 1990. Inventario fitosociológico del pastizal de la depresión de Salado (Prov. Bs. As.) en un área representativa de sus principales ambientes. Darwiniana, 30:27-69.

Burrough, P; P van Gaans \& R MacMillan. 2000. Highresolution landform classification using fuzzy k-means. Fuzzy Sets and Systems, 113:37-52.

Deregibus, V \& M Cahuepé. 1983. Pastizales naturales de la Depresión del Salado: utilización basada en conceptos ecológicos. Revista de Investigaciones Agropecuarias. Buenos Aires. Argentina. INTA XVIII, 1:47-78.

DoKuchaev, V. 1886. Razbor glavneishich pochvennych ponyatiy (in Russian). En: Dokuchaev, VV (ed.). Selected Papers 1949. Moscow. Selhozgiz, 3:161-239.

GoEtz, S. 1997. Multi-sensor analysis of IVN, surface temperature and biophysical variables at a mixed grassland site. International Journal of Remote Sensing, 18:71-94.

HotelLiNG, H. 1936. Relations between two sets of variates. Biometrika, 28:321-377.

INTA. 1977-1989. Mapas de suelo de la provincial de Buenos Aires escala 1:50.000. Eds. INTA.

INTERNATIONAL BANK FOR RECONSTRUCTION AND DEVELOPMENT. 1967. "Balcarce" livestock development project - Argentina. Report No TO-540a. Pp. 55.

JÖNSSON, P \& L EKLUNDH. 2004. TIMESAT—a program for analyzing time-series of satellite sensor data. Computers $\mathcal{E}$ Geoscience, 30:833-845.

León, R; S Burkart \& C Movia. 1979. Relevamiento fitosociológico del pastizal del norte de la depresión del Salado: La vegetación de la república Argentina. Serie fitogeográfica $n^{\circ} 17$. Buenos Aires. INTA, Pp. 90.

Masuelli, S; P Vázquez \& O Faure. 2006. Adecuación del DEM del STRM de 90 metros para usos hidrológicos en llanuras. XII Simposio internacional SELPER: SIG y Percepción Remota aplicados a "Riesgos Naturales y Gestión del Territorio, 24 al 29 septiembre.

Mattar, C; J Sobrino; Y Julien; B Franch \& R Oltra. 2008. Método simple para identificación de zonas homogéneas de IVN y temperatura de superficie en la Península Ibérica.
Revista de Teledetección, 30:92-101.

Mc QueEn, J. 1967. Some Methods for classification and Analysis of Multivariate Observations. Proceedings of 5-th Berkeley Symposium on Mathematical Statistics and Probability, Berkeley, University of California Press, 1 : 281-297.

Miaczynski, C. 1995. Los suelos hidromórficos e hidrohalomórficas de la provincia de Buenos Aires. Rev. Fac. Agr.,15:23-36.

NASA-NGA. 2000. The Shuttle Radar Topography Mission. www2.jpl.nasa.gov/srtm/.

Nouvellon, Y; S Moran; D Seen; R Bryant; S Rambal; et AL. 2001. Coupling a grassland ecosystem model with Landsat imagery for a 10-year simulation of carbon and water budgets. Remote Sensing of Environment, 78: 131-149.

Perelman, S; R León \& M Oesterheld. 2001. Cross scale vegetation patterns of flooding Pampa grasslands. Journal of Ecology, 89:562-577.

Potтer, C \& V BROокs. 1998. Global analysis of empirical relations between annual climate and seasonality of IVN. International Journal of Remote Sensing, 19:2921-2948.

Rouse, J; R HaAs; J Schell; D Deerino \& J Harlan. 1974. Monitoring the vernal advancement of retrogradation of natural vegetation. NASA/OSFC. Type III. Final report. Oreenbello MD. Pp. 371.

SAVITZKY, A \& M Golay. 1964. Smoot hi ng and differentiation of data by simplified least squares procedures. Analytical Chemistry, 36:1627-1639.

TACHIKaWA, T; M KaKU \& A IWASAKI. 2011. ASTER GDEM Version 2 Validation. Presentation at the 39th ASTER Science Team Meeting, Tokyo, Japan.

Tiezen, L; B Reed; N Bliss; D Dejong \& B Wylie. 1997. IVN, $\mathrm{C} 3$ and $\mathrm{C} 4$ production, and distributions in great plains grassland land cover classes. Ecological applications, 7: 59-78.

Turner, M \& R GARDNER (EDS.). 1991: The Analysis and Interpretation of Landscape Heterogeneity. Pp. 536 en: Quantitative Methods in Landscape Ecology. Ecological Studies v. 82. Springer-Verlag, New York, USA.

SoIl Survey Staff. 2010. Keys of soil taxonomy. $11^{\text {th }}$ edition. Eds. USDA- NRCS. Pp. 374.

VÁZOUEZ, P; I ENTRAigas; M VARNi; M Gandini \& E Usunoff. 2003. Identificación de patrones de anegamiento en la cuenca del arroyo del Azul mediante el uso de imágenes LANDSAT. Revista de Teledetección, 19:43-49.

VÁzQuez, P; F Cabria; M Rojas \& M CALANDRONi. 2009. Riesgo de anegamiento: estimaciones para la cuenca baja del río Salado. Asociación Argentina de la Ciencia de Suelo, 27:237-246.

VÁzquez, P; M Rojas \& J Burges. 2008. Caracterización y tendencias de la ganadería bovina en la cuenca del Salado. Veterinaria Argentina, 25:572-584.

Vervoorst, F. 1967. Las comunidades vegetales de la depresión del Salado. La vegetación de la república Argentina. Serie Fitogeográfica $\mathrm{N}^{\circ} 7$. Buenos Aires. INTA. Pp. 259.

Zhu, A; B Hudson; J Burt; K Lubich \& D Simonson. 2001. Soil mapping using GIS, expert knowledge, and fuzzy logic. Soil Sci. Soc. Am. J., 65:1463-1472. 